\title{
ON THE NATURE OF WEAK SOLUTIONS AND SOME ABSTRACT CAUCHY PROBLEMS
}

\author{
BY ROBERT CARROLL'
}

Communicated by F. Browder, June 13, 1966

We give here some "intrinsic" results about abstract linear Cauchy problems in Hilbert space. It is first shown that weak solutions are really strong solutions of another problem and this leads to a new type of uniqueness theorem for weak solutions. Then the operational nature of certain standard hypotheses in existence theory is exhibited and an abstract existence theorem in linked operators is stated. The details will appear in [5].

1. Let $H$ be a Hilbert space and $V(t)$ a measurable family of Hilbert spaces for Lebesgue measure (cf. Dixmier [7]) with $V(t) \subset H$ algebraically and topologically and dense in $H$. Let $W=\int \oplus V(t) d t, 0 \leqq t$ $\leqq T<\infty$ with scalar product $((u, v))=\int_{0}^{T}((u(t), v(t)))_{t} d t$. For each $t \in[0, T]$ let $a(t$,$) denote a continuous sesquilinear form on$ $V(t) \times V(t)$ and let $A(t) \in L(V(t), V(t))$ be the associated continuous linear operator defined by $a(t, x, y)=((A(t) x, y))$ for $x, y \in V(t)$. Suppose further that the field of operators $A(t)$ is measurable (cf. [7]) and $|a(t, u, v)| \leqq M\|u\|_{t}\left\|_{v}\right\|_{t}$ with $M$ independent of $t$ for $u, v \in V(t)$. We shall consider functions $u \in W$ satisfying

$$
\text { (1) }-\int_{0}^{T}\left(u, v^{\prime}\right) d t+\int_{0}^{T} a(t, u, v) d t+\lambda \int_{0}^{T}(u, v) d t=\int_{0}^{T}(f, v) d t
$$

for all $v \in W$ with $v^{\prime} \in L^{2}(H)$ and $v(T)=0$. Here (, ) denotes the scalar product in $H$ and all derivatives are taken in $D^{\prime}(H)$ (cf. [15]); $f \in L^{2}(H)$ is supposed given. By the nature of differential problems of this type we can always add a $\lambda$ term as indicated with arbitrarily large $\lambda$ (cf. [2], [3], [10]). We are dealing with the case $u(0)=0$ for convenience only. In supposing $\operatorname{Re} a(t, u, u)+\lambda|u|^{2} \geqq C\|u\|_{t}^{2}$ for $u \in V(t)$ Lions proves existence in [10]. Under more hypotheses uniqueness theorems are given by Lions in [10], [11]. In the case that $V(t)=V$ is constant and $W$ is $L^{2}(V)$ the hypotheses already indicated are enough for existence and uniqueness (see Lions [10] - other proofs can be obtained by specializing Browder's more general nonlinear

\footnotetext{
1 Research supported in part by the National Science Foundation under Grant GP-4575.
} 
results [2], [3] to the linear case and similar theorems under various hypotheses can be found in Kato and Tanabe [9], Kato [8], Poulsen [13], Sobolevskij [16], and in many other articles too numerous to list here).

Now a subhilbert space $V(t)$ of $H$ as above can be described as the domain of a closed densely defined positive definite self adjoint "standard" operator $B^{1 / 2}(t)$ where $B^{1 / 2}(t)$ maps onto $H$ with a continuous inverse. In fact $B(t)=\theta L(t)^{-1}$ where $\theta: H^{\prime} \rightarrow H$ is the canonical anti-isomorphism and $L(t)$ is the Schwartz anti-kernel of $V(t)$ relative to $H$ (see Schwartz [14] for general notions and the author's paper [4] for proof). Here $V(t)$ is provided with the Hilbert structure $((u, v))_{t}=\left(B^{1 / 2}(t) u, B^{1 / 2}(t) v\right)$. (See [4].) If $V(t)$ has the graph Hilbert structure of an operator $E(t)$ (closed and densely defined) then $B(t)$ $=\left(1+E^{*}(t) E(t)\right)$ (see [4]). Next observe that if $x \in H, y \in V(t)$ then $y \rightarrow(x, y)_{H}$ determines an antilinear form on $V(t)$ which we write $((J x, y))_{i}$. Therefore $\left(B^{1 / 2} J x, B^{1 / 2} y\right)=(x, y)$ and consequently $J=B^{-1}$ (we omit $t$ occasionally for simplicity in writing). Now (1) can be rewritten in the form

$$
-\int_{0}^{T}\left(u, v^{\prime}\right) d t=\int_{0}^{T}((\xi, v))_{t} d t
$$

where $\xi=J(t) f(t)-\lambda J(t) u(t)-A(t) u(t)$ is a function.

Now in general $u^{\prime}$ will not be a function (cf. [9]) but we will show that $\left(B^{-1 / 2} u\right)^{\prime}$ is a function and reduce the weak problem to a strong problem involving an additional term measuring the way in which the scalar product changes. If $V(t)$ is constant this is related to constructions in [1], [3]. It is to be emphasized here that hypotheses of differentiability on $B^{-1 / 2}(t)$ can now be interpreted directly as smoothness conditions on the way $V(t)$ changes. Thus our formulation will be intrinsic in a certain sense.

Let $D_{w}=\left\{\phi=B^{-1 / 2}(t) \psi, \psi \in D(H)\right\}$ and assume $B^{-1 / 2}$ is strongly $C^{1}$ in the sense that $B^{-1 / 2}(t) x$ is $C^{1}$ for $x \in H$ with $\left(B^{-1 / 2} x\right)^{\prime}=\left(B^{-1 / 2}\right)^{\prime} x$ for $\left(B^{-1 / 2}\right)^{\prime} \in L(H)=L(H, H)$. Then by Banach-Steinhaus $\left\|B^{-1 / 2}(t)\right\|$ $\leqq M$ for $0 \leqq t \leqq T$ and $\phi$ will be $C^{1}\left(C^{1}\right.$ means continuously differentiable). Thus $\phi \in D_{w}$ is admissible as $v$ in (2) (note $\phi^{\prime} \in W$ necessarily). Now writing $\left(B^{-1 / 2}\right)^{\prime}=\dot{B}^{-1 / 2}$ for simplicity, $\phi=B^{-1 / 2} \psi$, we know $\phi^{\prime}$ $=\dot{B}^{-1 / 2} \psi+B^{-1 / 2} \psi^{\prime}$. Then from (2)

$$
-\int_{0}^{T}\left(B^{-1 / 2} u, \psi^{\prime}\right) d t-\int_{0}^{T}\left(B^{-1 / 2} u, \psi\right) d t=\int_{0}^{T}((\xi, \phi))_{t} d t
$$

since $\dot{B}^{-1 / 2}$ will be self adjoint. This means 


$$
\left\langle\left(B^{-1 / 2} u\right)^{\prime}, \psi\right\rangle-\left\langle B^{-1 / 2} u, \psi\right\rangle=\left\langle B^{1 / 2} \xi, \psi\right\rangle
$$

where $\langle$,$\rangle means D(H), D^{\prime}(H)$ conjugate linear duality and $\left(B^{-1 / 2} u\right)^{\prime}$ means the distribution derivative. Hence in $D^{\prime}(H)\left(B^{-1 / 2} u\right)^{\prime}$ is a function and we have in $L^{2}(H)$

$$
\left(B^{-1 / 2} u\right)^{\prime}-B^{-1 / 2} u+\lambda B^{-1 / 2} u+B^{1 / 2} A u=B^{-1 / 2} f .
$$

Theorem 1. Let $A(t) \in L(V(t), V(t))$ be a measurable family of operators associated with the continuous sesquilinear forms $a(t, u, v)$ where $|a(t, u, v)| \leqq M\|u\|_{t}\left\|_{v}\right\|_{t}, M$ constant, and let $B^{1 / 2}(t)$ be the standard operator for $V(t)$. Let $u$ be a solution of the weak problem (1) and assume $B^{-1 / 2}(t)$ is strongly $C^{1}$ as indicated. Then $u$ is a solution of the strong problem (5) with $u(0)=0$.

REMARK 1. As an example consider the case where $a(t, u, v)$ $=\left(E(t) u, E^{*}(t) v\right)$ and say $E(t)=F(t)^{1 / 2}$ as a most natural case. Suppose for example that $D(E(t)) \subset D\left(E^{*}(t)\right)$ with $\left|E^{*}(t) x\right|^{2} \leqq c\left(|E(t) x|^{2}\right.$ $\left.+|x|^{2}\right)$ for $x \in D(E(t))$ and set $V(t)=D(E(t))$. Then $B(t)=1$ $+E^{*}(t) E(t)$ and $a(t, u, v)=\left(B^{1 / 2} B^{-1 / 2} K^{*} E u, B^{1 / 2} u\right)$ where $K=E^{*} B^{-1 / 2}$ $\in L(H)$. Then $A=B^{-1 / 2} K^{*} E$ and in the event that $u \in D(F)$ one has in fact $A u=B^{-1} F u$ and the term $B^{1 / 2} A u$ in (5) becomes $B^{-1 / 2} F u$. This is the situation when $u$ is a strong solution of $u^{\prime}+F(t) u=f$.

2. Now we show that knowing how to write a weak problem as a strong solution of the type (5) leads to a new kind of uniqueness theorem for weak solutions. We do not attempt to compare hypotheses here with those of Lions [11] since the formulation of our hypotheses is different; this will be examined in [5]. We remark however that the hypotheses are realistic. First, setting $f=0$ in (5), we take scalar products in $H$ with $B^{-1 / 2} u$ to obtain

$$
\begin{aligned}
\frac{1}{2} \frac{d}{d t}\left|B^{-1 / 2} u\right|^{2}+\lambda\left|B^{-1 / 2} u\right|^{2} & +\operatorname{Re}\left(B^{1 / 2} A u, B^{-1 / 2} u\right) \\
& -\operatorname{Re}\left(B^{-1 / 2} u, B^{-1 / 2} u\right)=0 .
\end{aligned}
$$

Now one need only make suitable hypotheses on the last two terms and these can be motivated by Remark 1 . We will give details of this in [5]. For example, recalling that $\lambda$ may be assumed arbitrarily large, one has

THEOREM 2. Let the hypotheses of Theorem 1 hold with $\operatorname{Re}(A u, u)$ $\geqq-\gamma\left|B^{-1 / 2} u\right|^{2}$ and $\left|\left(\dot{B}^{-1 / 2} u, B^{-1 / 2} u\right)\right| \leqq C\left|B^{-1 / 2} u\right|^{2}$ for $u(t) \in V(t)$. Then solutions of (1) are unique. 
REMARK 2. The last condition holds for example if $\dot{B}^{-1 / 2} B^{1 / 2}$ determines a bounded operator in $H$. We note that the condition on $\operatorname{Re}(A u, u)$ is not a straight monotonicity requirement since that involves usually $\operatorname{Re} a(t, u, u)=\operatorname{Re}\left(B^{1 / 2} A u, B^{1 / 2} u\right)$.

3. We want to indicate briefly here some further results toward the solution of the problem $S u=L u+A u=f$ with $L$ and $A$ closed, densely defined, linear operators in a Hilbert space $H$ (see [6]). Thus for example (see [5] for details).

TheOREM 3. Let $A=A_{1}+A_{2}$ with $A_{1}$ self adjoint, positive, and onto; $D\left(A_{1}\right)=D(A) \subset D\left(A_{2}\right) ;\left\|A_{2} u\right\| \leqq \beta\left\|A_{1} u\right\|+c_{2}\left\|A_{1}^{1 / 2} u\right\|$ for $u \in D\left(A_{1}\right) ; L$ and $A$ be closed, densely defined, and linear with $L^{*}$ accretive; and suppose $\left|\operatorname{Re}\left(A_{1}^{-1 / 2} x, R x\right)\right| \leqq \alpha\|x\|^{2}+c_{1}\left\|A_{1}^{-1 / 2} x\right\|^{2}$ for $x \in D\left(L^{*}\right)$ where $R=L^{*} A_{1}^{-1 / 2}-A_{1}^{-1 / 2} L^{*}$ and it is assumed that $x \in D\left(L^{*}\right)$ implies $A_{1}^{-1 / 2} x \in D\left(L^{*}\right)$. Assume $\alpha+\beta<1$ and that $\eta$ is chosen small enough so that $\gamma=1-\alpha-\beta-c_{2} \eta / 2>0$. Then for $k \geqq c_{1}+c_{2} 2 \eta$ the operator $L+A+k$ maps onto $H$.

REMARK 3. This theorem is in a sense copied from a technique of Lions [10]. However there are several important differences. First, as indicated partially in [6], we show monotonicity can be used, and, in another version we reveal what the usual hypotheses on $-R$ $=(d / d t) F_{1}(t)^{-1}$ really mean (when $A$ arises from $F(t)=F_{1}(t)+F_{2}(t)$ ); note that $R$ is related to this operator by $\widetilde{R}=A_{1}^{-1 / 2} R+R A_{1}^{-1 / 2}$. Also it should be pointed out here how much of the work $A$ does; monotonicity of $L$ is not assumed. Theorem 1 of [6, part II], can also be generalized out of the tensor product situation (see [5]).

\section{REFERENCES}

1. C. Baiocchi, Regolarita e unicita della soluzione di una equazione differenziale astratta, Rend. Sem. Mat Padova 35 (1965), 380-417.

2. F. Browder, Non-linear equations of evolution, Ann. of Math. 80 (1964), 485-523.

3. - Non-linear initial value problems, Ann. of Math. 82 (1965), 51-87.

4. R. Carroll, On the structure of some abstract differential problems, Ann. Mat. Pura Appl. 72 (1966), 305-318.

5. - On the propagator equation, (to appear).

6. - Problems on linked operators. I, II, Math. Ann. 151 (1963), 272-282; 160 (1965), 233-256.

7. J. Dixmier, Les algébres d'opèrateurs dans l'espace Hilbertien, Gauthier-Villars, Paris, 1957.

8. T. Kato, Non-linear evolution equations in Banach spaces, Proc. Sympos. Appl. Math. Vol. 17, Amer. Math. Soc., Providence, R. I., 1965, pp. 50-67.

9. T. Kato and H. Tanabe, On the abstract evolution equation, Osaka Math. J. 14 (1962), 107-133. 
10. J. L. Lions, Equations différentielles-opérationnelles, Springer, Berlin, 1961.

11. - Rémarques sur les équations différentielles opérationnelles, Osaka Math. J. 15 (1963), 131-142.

12. - Equations differentielles-operationelles dans les espaces de Hilbert, CIME, Varenna, 1963.

13. E. Poulsen, Evolutionsgleichungen in Banach-Räumen, Math. Z., 90 (1965), 286-309.

14. L. Schwartz, Sous-espaces Hilbertiens d'espaces vectoriels topologiques et noyaux associés (noyaux reproduisants), J. Analyse Math. 13 (1964), 115-256.

15. - Theorie des distributions d valeurs vectorielles, Ann. Inst. Fourier Grenoble (7) (8) (1957, 1958), 1-141, 1-209.

16. P. Sobolevskij, On equations of parabolic type in a Banach space, Trudy Moscov Mat. Obšc. 10 (1961), 297-350.

UNIVERSITY OF ILLINOIS 